\title{
GROUPS WHICH HAVE A FAITHFUL REPRESENTATION OF DEGREE LESS THAN $(p-1 / 2)$
}

\author{
WALTER Feit AND John G. Thompson
}

1. Introduction. Let $G$ be a finite group which has a faithful representation over the complex numbers of degree $n$. H. F. Blichfeldt has shown that if $p$ is a prime such that $p>(2 n+1)(n-1)$, then the Sylow $p$-group of $G$ is an abelian normal subgroup of $G$ [1]. The purpose of this paper is to prove the following refinement of Blichfeldt's result.

THEOREM 1. Let $p$ be a prime. If the finite group $G$ has a faithful representation of degree $n$ over the complex numbers and if $p>2 n+1$, then the Sylow p-subgroup of $G$ is an abelian normal subgroup of $G$.

Using the powerful methods of the theory of modular characters which he developed, R. Brauer was able to prove Theorem 1 in case $p^{2}$ does not divide the order of $G$ [2]. In case $G$ is a solvable group, N. Ito proved Theorem 1 [4]. We will use these results in our proof.

Since the group $S L(2, p)$ has a representation of degree $n=(p-1) / 2$, the inequality in Theorem 1 is the best possible.

It is easily seen that the following result is equivalent to Theorem 1.

THEOREM 2. Let $A, B$ be $n$ by $n$ matrices over the complex numbers. If $A^{r}=I=B^{s}$, where every prime divisor of $r s$ is strictly greater than $2 n+1$, then either $A B=B A$ or the group generated by $A$ and $B$ is infinite.

For any subset $S$ of a group $G, C_{\theta}(S), N_{G}(S),|S|$ will mean respectively the centralizer, normalizer and number of elements in $S$. For any complex valued functions $\zeta, \xi$ on $G$ we define

$$
(\zeta, \xi)_{\theta}=\frac{1}{|G|} \sum_{G} \zeta(x) \overline{\xi(x)}
$$

and $\|\zeta\|_{\theta}^{2}=(\zeta, \zeta)_{\theta}$. Whenever it is clear from the context which group is involved, the subscript $G$ will be omitted. $H \triangleleft G$ will mean that $H$ is a normal subgroup of $G$. For any two subsets $A, B$ of $G, A-B$ will denote the set of all elements in $A$ which are not in $B$. If a subgroup of a group is the kernel of a representation, then we will also say that it is the kernel of the character of the given representation. All groups

Received November 25, 1960. The first author was partly supported by O. O. R. and an NSF Grant. The second author was partly supported by the Esso Research Foundation. 
considered are assumed to be finite.

2. Proof of Theorem 1. We will first prove the following preliminary result.

Lemma 1. Assume that the Sylow p-group $P$ of $N$ is a normal subgroup of $N$. If $x$ is any element of $N$ such that $C_{N}(x) \cap P=\{1\}$, then $\lambda(x)=0$ for any irreducible character $\lambda$ of $N$ which does not contain $P$ in its kernel.

Proof. Since $\left|C_{N}(x)\right|$ is not divisible by $p$, it is easily seen that $C_{N}(x)$ is mapped isomorphically into $C_{N / P}(\bar{x})$, where $\bar{x}$ denotes the image of $x$ in $N / P$ under the natural projection. Let $\mu_{1}, \mu_{2}, \cdots$ be all the irreducible characters of $N$ which contain $P$ in their kernel and let $\lambda_{1}, \lambda_{2}, \cdots$ be all the other irreducible characters of $N$. The orthogonality relations yield that

$$
\sum_{i}\left|\mu_{i}(x)\right|^{2}=\left|C_{N / P}(\bar{x})\right| \geqq\left|C_{N}(x)\right|=\sum_{i}\left|\mu_{i}(x)\right|^{2}+\sum_{i}\left|\lambda_{i}(x)\right|^{2} .
$$

This implies the required result.

From now assume that $G$ is a counter example to Theorem 1 of minimal order. We will show that $p^{2}$ does not divide $|G|$, then Brauer's theorem may be applied to complete the proof. The proof is given in a series of short steps.

Clearly every subgroup of $G$ satisfies the assumption of Theorem 1 , hence we have

(I) The Sylow p-group of any proper subgroup $H$ of $G$ is an abelian normal subgroup of $H$.

Let $P$ be a fixed Sylow $p$-group of $G$. Let $Z$ be the center of $G$.

(II) $P$ is abelian.

As $P$ has a faithful representation of degree $n<p$, each irreducible constituent of this representation has degree one. Therefore in completely reduced form, the representation of $P$ consists of diagonal matrices. Consequently these matrices form an abelian group which is isomorphic to $P$.

(III) $G$ contains no proper normal subgroup whose index in $G$ is a power of $p$.

Suppose this is false. Let $H$ be a normal subgroup of $G$ of minimum 
order such that $[G: H]$ is a power of $p$. Let $P_{0}$ be a Sylow $p$-group of $H$. By (I) $P_{0} \triangleleft H$, hence $P_{0} \triangleleft G$. Thus $C_{\theta}\left(P_{0}\right) \triangleleft G$. If $C_{\theta}\left(P_{0}\right) \neq G$, then by (I) and (II), $P \triangleleft C_{G}\left(P_{0}\right)$, thus $P \triangleleft G$ contrary to assumption. Therefore $C_{\theta}\left(P_{0}\right)=G$. Burnside's Theorem ([3], p. 203) implies that $H$ contains a normal $p$-complement which must necessarily be normal in $G$. The minimal nature of $H$ now yields that $p$ does not divide $|H|$.

If $q$ is any prime dividing $|H|$, then it is a well known consequence of the Sylow theorems that it is possible to find a Sylow $q$-group $Q$ of $H$ such that $P \subseteq N(Q)$. Hence $P Q$ is a solvable group which satisfies the hypotheses of Theorem 1. Ito's Theorem [4] now implies that $P \triangleleft P Q$, thus $Q \subseteq N(P)$. As $q$ was an arbitrary prime dividing $|H|$, we get that $|H|$ divides $|N(P)|$. Consequently $N(P)=G$, contrary to assumption.

(IV) $Z$ is the unique maximal normal subgroup of $G$. $G / Z$ is a noncyclic simple group. $|Z|$ is not divisible by $p$.

Let $H$ be a maximal normal subgroup of $G$, hence $G / H$ is simple. Let $P_{0}$ be a Sylow $p$-group of $H$. Then by (I) $P_{0} \triangleleft H$, hence $P_{0} \triangleleft G$, thus $C\left(P_{0}\right) \triangleleft G$. If $C\left(P_{0}\right) \neq G$, then by (I) and (II) $P \triangleleft C\left(P_{0}\right)$, hence $P \triangleleft G$ contrary to assumption. Therefore $C\left(P_{0}\right)=G$. If $P_{0} \neq\{1\}$, then it is a simple consequence of Grün's Theorem ([3], p. 214) that $G$ contains a proper normal subgroup whose index is a power $p$. This contradicts (III). Hence $P_{0}=\{1\}$ and $p$ does not divide $|H|$.

By (III) $P H \neq G$, hence by (I) $P \triangleleft P H$. Consequently $P H=P \times H$, and $P \subseteq C(H) \triangleleft G$. If $C(H) \neq G$, then (I) yields that $P \triangleleft C(H)$. Hence once again $P \triangleleft G$, contrary to assumption. Consequently $C(H)=G$. Therefore $H \subseteq Z$. As $G$ is not solvable, neither is $G / H$. Now the maximal nature of $H$ yields that $H=Z$ and suffices to complete the proof.

(V) $P \cap x P x^{-1}=\{1\}$ unless $x$ is in $N(P)$.

Let $D=P \cap x P x^{-1}$ be a maximal intersection of Sylow $p$-groups of $G$. Then $P$ is not normal in $N(D)$. Hence by (I) $N(D)=G$, or $D \triangleleft G$. However (IV) now implies that $D \cong Z$. Hence (IV) also yields that $D=\{1\}$ as was to be shown.

Define the subset $N_{0}$ of $N(P)$ by

$$
N_{0}=\{x \mid x \in N(P), C(x) \cap P \neq\{1\}\} .
$$

Clearly $\{P, Z\} \subseteq N_{0}$.

(VI) $N\left(N_{0}\right)=N(P) . \quad\left(N_{0}-Z\right) \cap x\left(N_{0}-Z\right) x^{-1}$ is empty unless $x \in N(P)$.

Clearly $N(P) \subseteq N\left(N_{0}\right)$. Since $P$ consists of all elements in $N_{0}$ whose 
order is a power of $p$, it follows that $N\left(N_{0}\right) \subseteq N(P)$.

Suppose $y \in\left(N_{0}-Z\right) \cap x\left(N_{0}-Z\right) x^{-1}$. Then $y$ and $x^{-1} y x$ are both contained in $\left(N_{0}-Z\right)$. Let $P_{0}=C(y) \cap P, P_{1}=C\left(x^{-1} y x\right) \cap P$. By assumption $P_{0} \neq\{1\} \neq P_{1}$. It follows from the definitions that $P_{0}$ and $x P_{1} x^{-1}$ are both contained in $C(y)$. Since $y$ is not in $Z, C(y) \neq G$. Hence (I) yields that $P_{0}$ and $x P_{1} x^{-1}$ generate a $p$-group. Thus by (II) $x P_{1} x^{-1} \subseteq C\left(P_{0}\right)$. Now (V) implies that $x P_{1} x^{-1} \leqq N(P)$. Consequently $x P_{1} x^{-1} \leqq P$. By $(\mathrm{V})$, this yields that $x \in N(P)$ as was to be shown.

From now on we will use the following notation:

$$
|P|=p^{e}, \quad|Z|=z, \quad|N(P)|={ }^{\prime} p^{e} z t .
$$

Let $\chi_{0}=1, \chi_{1}, \cdots$ be all the irreducible characters of $G$. Define $\alpha_{i}, \beta_{i}, b_{i}$ by

$$
\chi_{i_{\mid N(P)}}=\alpha_{i}+\beta_{i}, \quad b_{i}=\beta_{i}(1)
$$

where $\alpha_{i}$ is a sum of irreducible characters of $N(P)$, none of which contain $P$ in their kernel and $\beta_{i}$ is a character of $N(P)$ which contains $P$ in its kernel.

(VII) If $i \neq 0$, then $b_{i}<\left(1 / p^{e / 2}\right) \chi_{i}(1)$.

By (VI) $\left(N_{0}-Z\right)$ has $|G| / p^{e} z t$ distinct conjugates and no two of them have any elements in common. Since $\chi_{i}$ is a class function on $G$, this yields that

$$
\begin{aligned}
1 & =\left\|\chi_{i}\right\|^{2}>\frac{1}{|G|} \frac{|G|}{p^{e} z t} \Sigma_{\left(N_{0}-Z\right)}\left|\chi_{i}(x)\right|^{2} \\
& =\frac{1}{p^{e} z t}\left\{-\Sigma_{Z}\left|\chi_{i}(x)\right|^{2}+\Sigma_{N_{0}}\left|\alpha_{i}(x)+\beta_{i}(x)\right|^{2}\right\} .
\end{aligned}
$$

If $x \in Z$, then $\left|\chi_{i}(x)\right|^{2}=\left|\chi_{i}(1)\right|^{2}$. As $P \subseteq N_{0}$, we get that

$1>\frac{1}{p^{e} z t}\left[-\left|\chi_{i}(1)\right|^{2} z+\Sigma_{N_{0}}\left\{\left|\alpha_{i}(x)\right|^{2}+\alpha_{i}(x) \overline{\beta_{i}(x)}+\overline{\alpha_{i}(x)} \beta_{i}(x)\right\}+\Sigma_{P Z}\left|\beta_{i}(x)\right|^{2}\right]$.

Since $P$ is in the kernel of $\beta_{i}$, we get that $\left|\beta_{i}(x)\right|=b_{i}$ for $x \in P Z$. Lemma 1 implies that $\alpha$ vanishes on $N(P)-N_{0}$. Hence

$$
1>\frac{-\left|\chi_{i}(1)\right|^{2}}{p^{e} t}+\left\|\alpha_{i}\right\|_{N(P)}^{2}+\left(\alpha_{i}, \beta_{i}\right)_{N(P)}+\left(\overline{\left(\alpha_{i}, \beta_{i}\right.}\right)_{N(P)}+\frac{b_{i}^{2}}{t} .
$$

By definition $\left(\alpha_{i}, \beta_{i}\right)=0$, hence

$$
\frac{\left|\chi_{i}(1)\right|^{2}}{p^{e} t}>\left\|\alpha_{i}\right\|_{N(P)}^{2}-1+\frac{b_{i}^{2}}{t}
$$

By (IV) the normal subgroup generated by $P$ is all of $G$, hence $\alpha_{i} \neq 0$. 
Therefore $\left\|\alpha_{i}\right\|_{N(P)}^{2} \geqq 1$. This finally yields that

$$
\frac{\left|\chi_{i}(1)\right|^{2}}{p^{e} t}>\frac{b_{i}^{2}}{t},
$$

which is equivalent to the statement to be proved.

(VIII) If $\Gamma$ is the character of $G$ induced by the trivial character $1_{P}$ of $P$, then $\left(\Gamma, \chi_{i}\right)=b_{i}$.

If $\lambda$ is an irreducible character of $N(P)$ which does not contain $P$ in its kernel, then $\lambda$ is not a constituent of the character of $N(P)$ induced by $1_{P}$. Hence by the Frobenius reciprocity theorem $\left(\lambda_{\mid P}, 1_{P}\right)_{P}=\mathbf{0}$. Consequently $\left(\alpha_{i \mid P}, 1_{P}\right)_{P}=0$. The Frobenius reciprocity theorem now implies that

$$
\left(\chi_{i}, \Gamma\right)=\left(\chi_{i \mid P}, 1_{P}\right)_{P}=\left(\beta_{i \mid P}, 1_{P}\right)=b_{i} .
$$

From now on let $\chi$ be an irreducible character of minimum degree greater than one. Define the integers $a_{i}$ by

$$
a_{i}=\left(\chi_{i}, \chi \bar{\chi}\right) \text {. }
$$

(IX) $\chi(1)-1 \leqq \sum_{i \neq 0} a_{i} b_{i}$.

By (VIII)

$$
\begin{aligned}
a_{0} b_{0}+\sum_{i \neq 0} a_{i} b_{i} & =(\Gamma, \chi \bar{\chi})=\frac{\chi(1)^{2}}{p^{e}}+\frac{1}{p^{e} z t} \Sigma_{P-(1)} z t \chi \bar{\chi}(x) \\
& =\frac{1}{p^{e}} \Sigma_{P} \chi \bar{\chi}(x)=\left\|\chi_{\mid P}\right\|_{P}^{2} .
\end{aligned}
$$

By (II), $\chi_{\mid P}$ is a sum of $\chi(1)$ linear characters of $P$. Consequently

$$
a_{0} b_{0}+\sum_{i \neq 0} a_{i} b_{i} \geqq \chi(1) .
$$

As $\chi$ is irreducible, $a_{0}=1$. Clearly $b_{0}=1$. This yields the desired inequality.

We will now complete the proof of Theorem 1 .

It follows from (IX) that

$$
\chi(1)-1 \leqq \sum_{i \neq 0} a_{i} b_{i} .
$$

(VII) yields that

$$
\sum_{i \neq 0} a_{i} b_{i}<\frac{1}{p^{e / 2}} \sum_{i \neq 0} a_{i} \chi_{i}(1) .
$$


The definition of the integers $a_{i}$ implies that

$$
\sum_{i \neq 0} a_{i} \chi_{i}(1)=\chi(1)^{2}-1 .
$$

Combining these inequalities we get that

$$
\chi(1)-1<\frac{\chi(1)^{2}-1}{p^{e / 2}},
$$

or

$$
p^{e / 2}<\chi(1)+1 .
$$

By assumption $\chi(1)<(p-1) / 2$, hence

$$
p^{e / 2}<\chi(1)+1<p .
$$

This implies that $e<2$. Thus $e \leqq 1$.

R. Brauer's theorem [2] now yields that $P \triangleleft G$ contrary to assumption. This completes the proof of Theorem 1 .

\section{BIBLIOGRAPHY}

1. H. F. Blichfeldt, On the order of linear homogeneous groups, Trans. Amer. Math. Soc., 4 (1903), 387-397.

2. R. Brauer, On groups whose order contains a prime number to the first power II, Amer.

J. of Math., 64 (1942), 421-440.

3. M. Hall, The Theory of Groups, The MacMillan Company, New York, 1959.

4. N. Ito, On a theorem of H. F. Blichfeldt, Nagoya Math. J., 5 (1954), 75-77.

CoRNELl UNIVERSity AND THE UNIVERSITY OF CHICAGO

HARVARD UNIVERSITY AND THE UNIVERSITY OF CHICAGO 